\title{
THEORY AND DESIGN OF PERFECT RECONSTRUCTION TRANSMULTIPLEXERS AND THEIR RELATION TO PERFECT RECONSTRUCTION QMF BANKS
}

\author{
R. D. Koilpillai, T. Q. Nguyen ${ }^{\ddagger}$ and P. P. Vaidyanathan
}

Dept. of Electrical Engineering, California Institute of Technology Pasadena, CA 91125

\begin{abstract}
The theory of transmultiplexers involves the design of filters for interconversion between Time Domain Multiplexing (TDM) and Frequency Division Multiplexing (FDM), such that the undesirable Crosstalk is minimized. In TDM $\rightarrow$ FDM $\rightarrow$ TDM conversion, the perfect reconstruction transmultiplexer (PR-TMUX) achieves complete Crosstalk Cancellation (CC) and is distortion-free. In this paper, we present an analysis of the PR-TMUX based on the polyphase component matrices of the filter banks used in TDM $\rightarrow$ FDM and FDM $\rightarrow$ TDM conversion respectively. Using that, a necessary and sufficient condition for complete $\mathrm{CC}$ is obtained. The close relation between PR-TMUX filters and PR-QMF banks is used to obtain a direct design procedure for PRTMUX filters.
\end{abstract}

\section{INTRODUCTION}

In recent years there has been a trend towards increasing use of digital techniques - Time Division Multiplexing (TDM) over the conventional analog Frequency Division Multiplexing (FDM) techniques [8], but it is expected that both will coexist for many years. In order to interface the two, we have to deal with TDM $\leftrightarrow$ FDM interconversion, which necessitates the Transmultiplexer. There exists a considerable amount of literature covering the theory, design and practical implementation of transmultiplexers [3-6], [8], [14], [15].

A schematic of the digital transmultiplexer system [14] is presented in Fig.1(a). $\left[x_{0}(n), x_{1}(n), \cdots x_{M-1}(n)\right]$ are the $M$ input signals (which are also the $M$ components of the TDM signal). $\left[F_{0}(z), F_{1}(z), \cdots F_{M-1}(z)\right]$ are the filters used in TDM $\rightarrow$ FDM conversion and will be called synthesis filters. The $M$ input signals are interpolated and passed through the synthesis filter bank and combined to produce the FDM

\footnotetext{
† Work supported in part by the National Science Foundation under Grants DCI 8552579 and MIP 8604456.

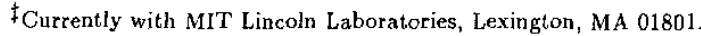

signal. At the other end, $\left[H_{0}(z), H_{1}(z), \cdots H_{M-1}(z)\right]$ are the filters used in FDM $\rightarrow$ TDM conversion and will be called analysis filters. The FDM signal $y(n)$ is passed through the analysis filter bank and then decimated to get back the TDM signals, $\hat{x}_{i}(n), 0 \leq i \leq M-1$.

Fig. 2(a) and Fig. 2(b) show the frequency spectra of a typical input $x_{i}(n)$ and the FDM signal $y(n)$ respectively. The voiceband channels are placed adjacent to one another and hence the bandwidth of the FDM signal is equal to the sum of the bandwidths of the component signais. Since the synthesis and analysis filters are non-ideal, there is leakage of signal from one voiceband channel to the others in the TDM $\rightarrow$ FDM $\rightarrow$ TDM conversion. This is defined as Crosstalk. In conventional transmultiplexer design [2], [8], in order to achieve crosstalk suppression, the filter specifications (stopband attenuation and transition bandwidth) are very stringent. To meet these specifications, many of the popular transmultiplexer designs use IIR filters [4]. However, there is a drawback in using IIR filters [8]. On the other hand, if we use FIR filters, the order of the filters has to be very high (typically $\approx 2000$ ) [2]. The high filter order implies increased number of computations and also a large overall group delay (which is large even if we use minimum phase FIR filters). So it is of considerable interest to obtain transmultiplexer designs, with FIR filters, which meet the crosstalk suppression specifications but have lower filter order than the conventional designs.

In this paper, we present new results on a method for efficient transmultiplexer design. This method, originally presented in [14], [15], focuses on Cancellation of Crosstalk. This means that the output signal is completely free from crosstalk. This should however be contrasted with the conventional approaches to transmultiplexer design, which aim to suppress crosstalk.

In this paper, we present the derivation of a necessary and sufficient condition for crosstalk cancellation, based on the polyphase component matrices of the analysis and synthesis filters. This condition is equivalent to the one derived in [15]. The new approach will throw additional light on the the 
understanding of the transmultiplexer problem. The main features are

1. Crosstalk Cancellation (CC).

2. Elimination of amplitude and phase distortions, i.e., exact recovery of the signals in TDM $\rightarrow$ FDM $\rightarrow$ TDM conversion.

If both the above conditions are satisfied, it will be called Perfect Reconstruction Transmultiplexer (PR-TMUX). If only the first condition is satisfied, then it will be called Crosstalkfree Transmultiplexer (CF-TMUX). The derivation of the new results will also serve to bring out the close connection between the transmultiplexer problem and the QMF problem. Using this, we obtain a direct design procedure for PR-TMUX and CF-TMUX filters. Since both the transmultiplexer circuit and the QMF circuit involve their respective analysis and synthesis filters, the filters and matrices associated with the QMF circuit always have a prime notation associated with them (as in $H^{\prime}(z)$ ), while the filters and matrices associated with the transmultiplexer do not. Further, $\left\{H_{i}(z), F_{i}(z)\right\}$ is an abbreviation for "Transmultiplexer with $M$ analysis filters $H_{i}(z)$ and $M$ synthesis filters $F_{i}(z), \quad 0 \leq i \leq M-1$." Similarly, $\left\{H_{i}^{\prime}(z), F_{i}^{\prime}(z)\right\}$ refer to the analysis and synthesis filters of the QMF circuit of Fig. 1(b).

\section{TRANSMULTIPLEXER ANALYSIS}

\subsection{Simplified Equivalent of the Transmultiplexer}

Using the polyphase decompositions of Types 1 and 2, [1], \{9], we can express the analysis and synthesis filter banks of the transmultiplexer circuit of Fig. 1(a) as

$$
\begin{array}{ll}
H_{k}(z)=\sum_{\ell=0}^{M-1} z^{-\ell} E_{k \ell}\left(z^{M}\right), & 0 \leq k, \ell \leq M-1 ;(1) \\
F_{k}(z)=\sum_{\ell=0}^{M-1} z^{-(M-1-\ell)} R_{\ell k}\left(z^{M}\right), & 0 \leq k, \ell \leq M-1 .(2)
\end{array}
$$

This representation leads to Fig. 3 where the polyphase component matrices $\mathbf{E}(z)$ and $\mathbf{R}(z)$ are defined as $\mathbf{E}(z)=$ $\left\{E_{k \ell}(z)\right]$ and $\mathbf{R}(z)=\left[R_{\ell k}(z)\right]$, respectively. Applying the standard identities of multirate signal processing [10], the interpolators and decimators can be moved appropriately to yield Fig. 4. The FDM signal $y(n)$ can be considered as a 'time-multiplexed version' of the signals $y_{i}(n)$ in Fig. 4, since the interpolators and the delay chain on the synthesis side implement a time domain multiplexer. On the other hand, the delay chain and decimators on the analysis side implement a time domain demultiplexer. This observation can be used to simplify the circuit of Fig. 4, by the following result:

Property 2.I : Consider an input signal $u(n)$ being passed through an interpolator, a delay of $k$ units and a decimator as shown in Fig. 5. In the Z-transform domain the output $v(n)$ can be expressed in terms of the input as

$$
V(z)= \begin{cases}0, & \text { if } k \neq \text { multiple of } M, \\ z^{-k / M} U(z), & \text { if } k \text { is a multiple of } M .\end{cases}
$$

Consequently, from Fig. 4 we obtain Fig. 6, which is a simplified equivalent representation of the transmultiplexer system. It is important to note that this is a Linear Time Invariant (but multi-input, multi-output) system, even though time-varying components such as decimators and interpolators are present in the original representation (Fig. 1(a)).

\subsection{Necessary and sufficient condition for CC}

From Fig. 6, we can write

$$
\left[\begin{array}{c}
\widehat{X}_{0}(z) \\
\widehat{X}_{1}(z) \\
\vdots \\
\widehat{X}_{M-1}(z)
\end{array}\right]=\mathbf{E}(z)\left[\begin{array}{cc}
0 & 1 \\
z^{-1} \mathbf{I}_{M-1} & 0
\end{array}\right] \mathbf{R}(z)\left[\begin{array}{c}
X_{0}(z) \\
X_{1}(z) \\
\vdots \\
X_{M-1}(z)
\end{array}\right] .
$$

To cancel crosstalk, it is evidently necessary and sufficient that

$$
\mathbf{E}(z)\left[\begin{array}{cc}
0 & 1 \\
z^{-1} \mathbf{I}_{M-1} & 0
\end{array}\right] \mathbf{R}(z)=\mathbf{C T}(z),
$$

where $\mathbf{T}(z)=\operatorname{diag}\left[T_{0}(z), T_{1}(z), \cdots T_{M-1}(z)\right]\left(T_{i}(z), 0 \leq\right.$ $i \leq M-1$, being stable transfer functions) and $\mathrm{C}$ is an arbitrary permutation matrix. If (5) is satisfied, we get a CF-TMUX. To simplify the notation, we shall restrict our attention to the $\mathrm{C}=\mathbf{I}$ case.

A special case of CF-TMUX (which has all $T_{i}(z)$ equal) is obtained where $\mathbf{T}(z)=S(z) \mathbf{I}_{M}$. In this case (5) becomes

$$
\mathbf{E}(z)\left[\begin{array}{cc}
\mathbf{0} & 1 \\
z^{-\mathbf{1}} \mathbf{I}_{M-1} & \mathbf{0}
\end{array}\right] \mathbf{R}(z)=S(z) \mathbf{I}_{M} .
$$

The condition on $\mathbf{R}(z)$ in terms of $\mathbf{E}(z)$ in order to achieve $\mathrm{CC}$ is

$$
\mathbf{R}(z)=S(z)\left[\begin{array}{cc}
0 & z \mathbf{I}_{M-1} \\
1 & 0
\end{array}\right] \mathbf{E}^{-1}(z)
$$

provided that $E^{-1}(z)$ is stable. From ( 7$)$ we see that $\mathbf{R}(z)$ may not be FIR even if $\mathrm{E}(z)$ is FIR, unless determinant of $\mathbf{E}(z)$ is a delay. Multiplying both sides of (7) by $\mathbf{E}(z)$, we get a necessary and sufficient condition for a CF-TMUX (which has all $T_{i}(z)$ equal)

$$
\mathbf{P}(z) \triangleq \mathbf{R}(z) \mathrm{E}(z)=S(z)\left[\begin{array}{cc}
0 & z \mathbf{I}_{M-1} \\
1 & 0
\end{array}\right] .
$$

In order for the CF-TMUX to achieve PR, it is necessary that $S(z)$ be a pure delay. So from (8) we can write the necessary and sufficient condition for a PR-TMUX as

$$
\mathbf{P}(z)=z^{-k}\left[\begin{array}{cc}
0 & z \mathbf{I}_{M-1} \\
1 & 0
\end{array}\right]=z^{-(k-1)}\left[\begin{array}{cc}
0 & \mathbf{I}_{M-1} \\
z^{-1} & 0
\end{array}\right] .
$$


where $k$ is a non-negative integer.

The next step is to establish the relationship between the QMF problem and the TMUX problem. From Lemma 3.2 [9] we know that a maximally decimated, M-channel QMF bank, with filters $\left\{H_{i}^{\prime}(z), F_{i}^{\prime}(z)\right\}$ gives rise to perfect reconstruction (PR) if and only if the matrix $\mathbf{P}^{\prime}(z)$, defined as $\mathbf{P}^{\prime}(z) \triangleq \mathbf{R}^{\prime}(z) \mathbf{E}^{\prime}(z)$, [where $\mathbf{E}^{\prime}\left(z^{M}\right)$ and $\mathbf{R}^{\prime}\left(z^{M}\right)$ are the polyphase component matrices of the analysis and synthesis filters respectively obtained as in (1), (2)] is of the form

$$
\mathbf{P}^{\prime}(z)=z^{-k_{1}}\left[\begin{array}{cc}
0 & \mathbf{I}_{M-n} \\
z^{-1} \mathbf{I}_{n} & 0
\end{array}\right], \quad 0 \leq n<M .
$$

where $k_{1}$ is a non-negative integer. In this case the overall transfer function $T(z)$ of the PR-QMF system is

$$
T(z)=z^{-(M-1+n)} z^{-k_{3} M} .
$$

The analysis and synthesis filters of a PR-QMF bank that satisfy equation (10) with $n=0$ will be referred to as a standard PR-QMF bank whereas if they satisfy equation (10) with $n \neq 0$, then they will be referred to as a n-skewed PR-QMF bank. We will now present two Lemmas, the first, relating the standard $\mathrm{PR}-\mathrm{QMF}$ and n-skewed $\mathrm{PR}$ QMF banks, and the second, relating the PR-TMUX filters and PR-QMF banks.

Lemma 2.1: A n-skewed PR-QMF bank $\left\{H^{\prime \prime}(z), F^{\prime \prime}(z)\right\}$ is always obtainable from a standard PR-QMF bank $\left\{H^{\prime}(z), F^{\prime}(z)\right\}$ by setting $H_{i}^{\prime \prime}(z)=H_{i}^{\prime}(z)$ and $F_{i}^{\prime \prime}(z)=z^{-n} F^{\prime}(z), \quad 0 \leq i \leq$ $M-1$.

Proof : It is given that

$$
\mathbf{P}^{\prime}(z)=\mathbf{R}^{\prime}(z) \mathbf{E}^{\prime}(z)=z^{-k_{1}} \mathbf{I}_{M} .
$$

We want to obtain $\left\{H_{i}^{\prime \prime}(z), F_{i}^{\prime \prime}(z)\right\}$ such that

$$
\mathbf{P}^{\prime \prime}(z)=\mathbf{R}^{\prime \prime}(z) \mathbf{E}^{\prime \prime}(z)=z^{-k_{1}}\left[\begin{array}{cc}
0 & \mathbf{I}_{M-n} \\
z^{-1} \mathbf{I}_{n} & 0
\end{array}\right] .
$$

Since $H_{i}^{\prime \prime}(z)=H_{i}^{\prime}(z), \quad \forall i$, we have $\mathbf{E}^{\prime \prime}(z)=\mathbf{E}^{\prime}(z)$, where $\mathbf{E}^{\prime}(z)$ and $\mathbf{E}^{\prime \prime}(z)$ are the polyphase component matrices of their respective analysis filter banks. So from (12) and (13), we can write

$$
\mathbf{R}^{\prime \prime}(z)=\left[\begin{array}{cc}
0 & \mathbf{I}_{M-n} \\
z^{-1} \mathbf{I}_{n} & 0
\end{array}\right] \mathbf{R}^{\prime}(z) .
$$

According to Type 2 polyphase decomposition, the synthesis filter bank $\mathbf{f}^{\prime}(z)$ corresponding to the polyphase component matrix $\mathbf{R}^{\prime}(z)$ can be represented as

$$
\mathbf{f}^{\prime}(z) \triangleq\left[\begin{array}{llll}
F_{0}^{\prime}(z) & F_{1}^{\prime}(z) & \cdots & F_{M-1}^{\prime}
\end{array}\right]^{T}=\mathbf{R}^{\prime T}\left(z^{M}\right) \mathbf{e}(z)
$$

where $\mathrm{e}(z)=\left[\begin{array}{llll}z^{-(M-1)} & z^{-(M-2)} & \ldots & 1\end{array}\right]^{T}$. Similarly we can write

$$
\mathrm{f}^{\prime \prime}(z)=\mathbf{R}^{\prime \prime T}\left(z^{M}\right) \mathbf{e}(z)
$$

$$
\begin{aligned}
& =\mathbf{R}^{\prime T}\left(z^{M}\right)\left[\begin{array}{cc}
0 & z^{-M} \mathbf{I}_{n} \\
\mathbf{I}_{M-n} & 0
\end{array}\right] \mathrm{e}(z) \\
& =z^{-n} \mathbf{R}^{\prime T}\left(z^{M}\right) \mathbf{e}(z)=z^{-n} \mathbf{f}^{\prime}(z) .
\end{aligned}
$$

In summary, we derived the relation between the synthesis filters of a standard PR-QMF bank and a n-skewed PR-QMF bank (with the analysis filters being fixed). Equivalently, the choice $F^{\prime \prime}(z)=F^{\prime}(z), H^{\prime \prime}(z)=z^{-n} H^{\prime}(z)$ will also enable us to obtain a n-skewed PR-QMF bank from a standard PRQMF bank.

$\nabla \nabla \nabla$

Lemma 2.2 : Let $\left\{H_{i}^{\prime}(z), F_{i}^{\prime}(z)\right\}$ represent a standard PRQMF bank. Define $H_{i}(z)=H_{i}^{\prime}(z), F_{i}(z)=z^{-1} F_{i}^{\prime}(z), \quad \forall i$. (Equivalently, the choice $H_{i}(z)=z^{-1} H_{i}^{\prime}(z), \quad F_{i}(z)=F_{i}^{\prime}(z)$ also works). Then $\left\{H_{i}(z), F_{i}(z)\right\}$ represents a PR-TMUX with $T_{i}\left(z^{M}\right)=z^{-1} T(z), \quad \forall i$.

Proof : For the standard PR-QMF bank,

$$
\mathbf{P}^{\prime}(z) \triangleq \mathbf{R}^{\prime}(z) \mathbf{E}^{\prime}(z)=z^{-k_{1}} \mathbf{I}_{M}
$$

From Lemma 2.1, we know that the particular choice of $\left\{H_{i}(z), F_{i}(z)\right\}$, as given in the statement of the Lernma, will yield $\mathbf{P}(z)$ in the form defined in equation (10) with $n=1$. So, for the TMUX filters, we can write

$$
\mathbf{P}(z)=\mathbf{R}(z) \mathbf{E}(z)=z^{-k_{1}}\left[\begin{array}{cc}
0 & \mathbf{I}_{M-1} \\
z^{-1} & 0
\end{array}\right] .
$$

From (20) we can write

$$
\mathbf{R}(z)=z^{-k_{1}}(z)\left[\begin{array}{cc}
0 & \mathbf{I}_{M-1} \\
z^{-1} & 0
\end{array}\right] \mathbf{E}^{-1}(z)
$$

Using (21) in (4), we get

$$
\widehat{X}_{i}(z)=z^{-\left(k_{1}+1\right)} X_{i}(z), \quad 0 \leq i \leq M-1 .
$$

Thus

$$
T_{i}\left(z^{M}\right)=z^{-1} T(z), \quad \forall i
$$

\section{Design procedure for PR-TMUX filters}

The results of the above two Lemmas, in addition to highlighting the close relationship between the PR-QMF bank and the PR-TMUX filters, clearly outline a design procedure to obtain PR-TMUX filters. The steps are :

1. Design a standard PR-QMF bank $\left\{H_{i}^{\prime}(z), F_{i}^{\prime}(z)\right\}$.

2. Choose the PR-TMUX filters to be $\left\{H_{i}^{\prime}(z), z^{-1} F_{i}^{\prime}(z)\right\}$ or $\left\{z^{-1} H_{i}^{\prime}(z), F_{i}^{\prime}(z)\right\}$

Extensive work has been done in the area of designing PRQMF banks [7], [9-13]. These results can be fully used in the design of PR-TMUX filters. 


\subsection{Alternate derivations of the $\mathrm{CC}$ conditions}

In [15], we find the following necessary and sufficient condition for $\mathrm{CC}$, in terms of the analysis and synthesis filters

$$
\mathbf{F}(z) \mathbf{H}(z)=\text { diag. }\left[\begin{array}{llll}
T_{0}\left(z^{M}\right) & T_{1}\left(z^{M}\right) & \cdots & T_{M-1}\left(z^{M}\right)
\end{array}\right] .
$$

where

$$
\mathbf{F}(z)=\left[\begin{array}{cccc}
F_{0}(z) & F_{0}(z W) & \cdots & F_{0}\left(z W^{M-1}\right) \\
F_{1}(z) & F_{1}(z W) & \cdots & F_{1}\left(z W^{M-1}\right) \\
\vdots & \vdots & \ddots & \vdots \\
F_{M-1}(z) & F_{M-1}(z W) & \cdots & F_{M-1}\left(z W^{M-1}\right)
\end{array}\right]
$$

and

$\mathbf{H}(z)=\left[\begin{array}{cccc}H_{0}(z) & H_{1}(z) & \cdots & H_{M-1}(z) \\ H_{0}(z W) & H_{1}(z W) & \cdots & H_{M-1}(z W) \\ \vdots & \vdots & \ddots & \vdots \\ H_{0}\left(z W^{M-1}\right) & H_{1}\left(z W^{M-1}\right) & \cdots & H_{M-1}\left(z W^{M-1}\right)\end{array}\right]$

From QMF theory [9], [15] we know that the Aliasing Cancellation (AC) equations can be written as

$$
\mathbf{H}(z) \mathbf{F}(z)=\operatorname{diag}\left[\begin{array}{llll}
T(z) & T(z W) & \ldots & T\left(z W^{M-1}\right)
\end{array}\right] .
$$

Note: The prime notation has not been used for the AC equation (24) since we are using the same analysis and synthesis filters in both the transmultiplexer circuit and the QMF circuit. So, from (23) and (24) AC and CC are simultaneously satisfied if and only if $T_{i}\left(z^{M}\right)=T(z), \quad \forall i$.

We will now show that this condition is equivalent to the one derived in the previous subsection.

Property 2.2 : Let $\left\{H_{i}^{\prime}(z), F_{i}^{\prime}(z)\right\}$ be an PR-QMF bank. Then the overall transfer function $T(z)$ of the PR-QMF system is a function of $z^{M}$ if and only if $\mathbf{P}^{\prime}(z)\left[=\mathbf{R}^{\prime}(z) \mathbf{E}^{\prime}(z)\right]$, has the form in equation (10) with $n=1$.

Lemma 2.3 : Let $\left\{H^{\prime}(z), F^{\prime}(z)\right\}$ be an PR-QMF bank. If the TMUX filters, $\{H(z), F(z)\}$, are chosen such that $H_{i}(z)=H_{i}^{\prime}(z)$ and $F_{i}(z)=F_{i}^{\prime}(z)$, then the TMUX is Crosstalkfree with all $T_{i}(z)$ equal if and only if $\mathbf{P}^{\prime}(z)\left[=\mathbf{R}^{\prime}(z) \mathbf{E}^{\prime}(z)\right]$, is of the form in equation (10) with $n=1$.

Proof : From (23) we have "TMUX is crosstalk free and all $T_{i}(z)$ are equal"

$$
\begin{array}{ll}
\Leftrightarrow & \mathbf{F}(z) \mathbf{H}(z)=T_{0}\left(z^{M}\right) \mathbf{I}_{M} \\
\Leftrightarrow & \mathbf{H}(z) \mathbf{F}(z)=T_{0}\left(z^{M}\right) \mathbf{I}_{M} . \\
\Leftrightarrow & T(z)=T_{0}\left(z^{M}\right), \text { [by using (26) in (24)]. }
\end{array}
$$

By Property 2.2, we conclude $\mathbf{P}(z)$ (and hence, $\mathbf{P}^{\prime}(z)$ ) has the form in equation (10) with $n=1$.

$\nabla \nabla \nabla$

\section{CONCLUSIONS}

In this paper, we have presented new results in the theory of Perfect Reconstruction Transmultiplexers (PR-TMUX). A necessary and sufficient condition for complete Crosstalk Cancellation (CC) is derived. The relation between PRQMF banks and PR-TMUX filters is shown. These results yield a design procedure for PR-TMUX filters.

\section{REFERENCES}

[1] M. G. Bellanger, G. Bonnerot and M. Coudreuse, "Digital filtering by polyphase network : application to sample rate alteration and filter banks," IEEE Trans. ASSP, pp109-114, April 1976.

[2] M. G. Bellanger, "On computational complexity in digital transmultiplexer filters," IEEE Trans. on Comm, pp 1461-1465, July 1982.

[3] Special issue on TDM-FDM conversion, IEEE Trans. on Comm, May 1978.

[4] Special issue on Transmultiplexers, IEEE Trans. on Comm, July 1982.

[5] R. E. Crochiere and L. R. Rabiner, "Multirate Digital Signal Processing," Englewood Cliffs, NJ: PrenticeHall, 1975.

[6] S. L. Freeny, "TDM/FDM translation as an application of digital signal processing," IEEE Comm Magazine, pp 5-15, January 1980.

[7] T. Q. Nguyen and P. P. Vaidyanathan, "Maximally decimated perfect reconstruction FIR filter banks with pairwise mirror-image analysis (and synthesis) frequency responses," IEEE Trans. ASSP, pp 693-706, May 1988.

[8] H. Scheuermann and H. Göckler, "A comprehensive survey of digital transmultiplexing methods," Proc. of the IEEE, pp 1419-1450, November 1981.

[9] P. P. Vaidyanathan, "Theory and design of $\mathrm{M}$-channel maximally decimated Quadrature Mirror Filters with arbitrary $M$, having the perfect reconstruction property," IEEE Trans. ASSP, pp 476-492, April 1987.

[10] P. P. Vaidyanathan, "Quadrature Mirror Filter Banks, M-band extensions and perfect reconstruction techniques." IEEE ASSP Magazine, vol. 4, pp 4-20, July $195 \overline{7}$.

[11] P. P. Vaidyanathan and P. Q. Hoang, "Lattice structures for optimal design and robust implementation of two-channel perfect reconstruction QMF banks, " IEEE Trans. ASSP, pp 81-94, January 1988.

[12] P. P. Vaidyanathan, "Multirate digital filters, filter banks, polyphase networks and applications : A tutorial," Proc. of the IEEE, to appear.

[13] P. P. Vaidyanathan, T. Q. Nguyen, Z. Doganata and T. Saramaki, "Improved technique for design of perfect reconstruction FIR QMF banks with lossless polyphase matrices," IEEE Trans. ASSP, pp 1042-1056, July 1989. 
[14] M. Vetterli, "Perfect transmultiplexers," IEEE Int. Conference on Acoustics, Speech and Signal Processing (ICASSP), Tokyo, April 1986, pp 2567-2570.

[15] M. Vetterli, "A theory of multirate filter banks," IEEE Trans. ASSP, pp 356-372, March 1987.

\section{FIGURES}

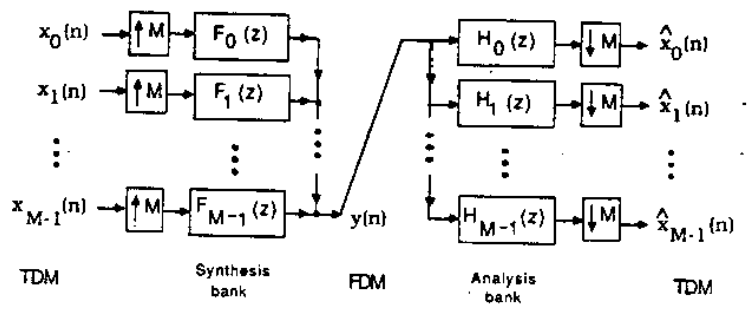

Fig. 1(a). The transmultiplexer system.

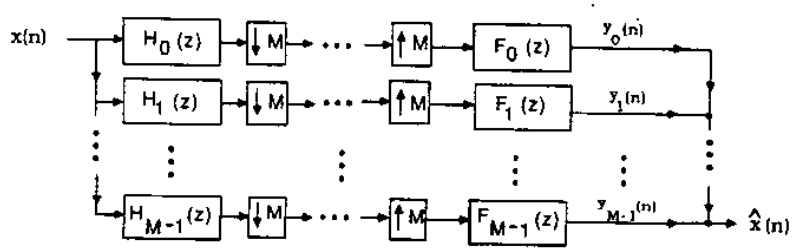

Fig. 1(b). The M-channel OMF circuit.

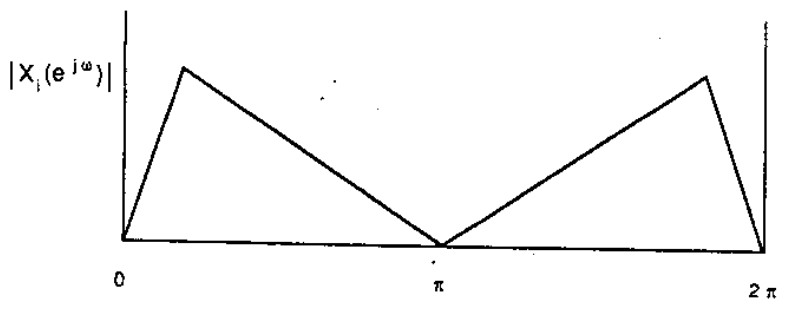

Fig. 2(a). Spectrum of input signal $x_{j}(n)$.

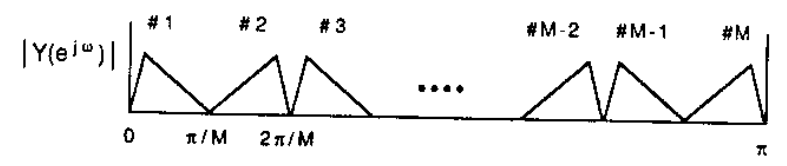

Fig. 2(b). Spectrum of the FDM signal $y(n)$.

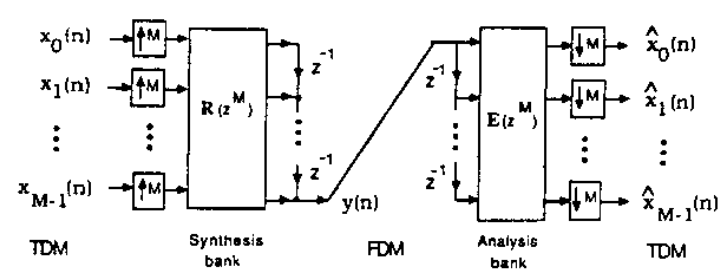

Fig. 3. The polyphase representation of the transmultiplexer system in Fig. 1.

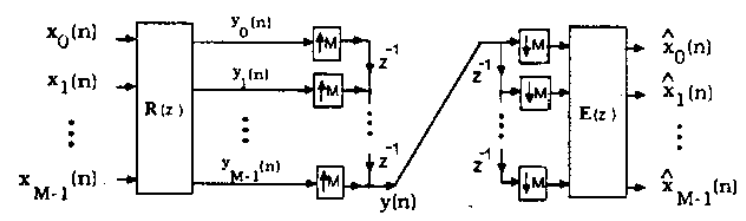

Fig. 4. Equivalent structure tor the transmultiplexer system in Fig. 3

$$
u(n) \rightarrow f M \rightarrow z^{-k} \rightarrow+M \rightarrow v(n)
$$

Fig. 5. A circuit with an interpolator, a delay and a decimator.

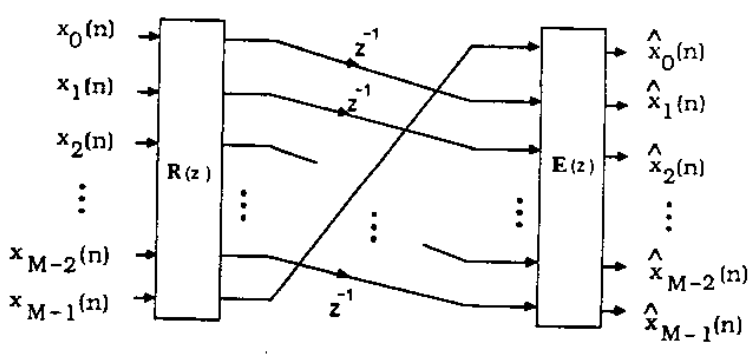

Fig. 6. The simplified equivalent representation of the transmultiplexer system in Fig. 1 . 